\title{
Persistance de Favositides microlamellaires (Cnidaria, Tabulata) dans le Dévonien
}

\section{Persistence of microlamellar Favositids (Cnidaria, Tabulata) in the Devonian}

\author{
Yves Plusquellec ${ }^{1}$ et Francis Tolirneur ${ }^{2}$ \\ ${ }^{1}$ Laboratoire de paléontologie et stratigraphie du Paléozoïque, UFR de sciences, université de Bretagne occidentale, 6, av. Victor-Le-Gorgeu, 29285 Brest \\ cedex, France \\ ${ }^{2}$ Unité de paléontologie et paléographie, université catholique de Louvain, 3, place Pasteur, 1348 Louvain-la-Neuve, Belgique
}

\begin{abstract}
The stmuctural and microstructural study of some devonian 'Favosites' shows the persistence of the microlamellar species related to Paleofavosites until the lower Eifelian. The evolution of the wall is characterized by an increase of the size of the microlamellae and the substitution of the granules of the median dark line by fiber-like units. (C) Académie des sciences/Elsevier, Paris.
\end{abstract}

Keywords: Favosites, Paleofavosites, Microlamellae, Algeria, Tabulata, Devonian

\section{RÉSUMÉ}

L'analyse structurale et microstructurale de quelques " Favosites " dévoniens montre la persistance des formes microlamellaires de type Paleofavosites jusqu'à l'Eifelien inférieur. L'évolution de la microstructure de la muraille se traduit par une augmentation de la taille des microlamelles et une fibrisation de la lame médiane. (C) Académie des sciences / Elsevier, Paris.

Mots clés : Favosites, Paleofavosites, Microlamelles, Algérie, Tabulata, Dévonien

\section{ABRIGED VERSION}

The genus Favosites Lamarck 1816 is supposed to be recorded all over the world from Ordovician to Permian. But Lafuste (1962) using $30 \mu \mathrm{m}$ thin sections, pointed out that the microstructure of the wall in Silurian and Devonian favositids is not identical. He is followed in that way especially by Smirnova (1974) and Mironova (1974).

The use of polished ultra-thin sections (the so-called 'LFP' in French) is an important step in the study of corals (Lafuste, 1970) as it allows us to see the outlines of each biocrystal and thus to identify correctly the microstructure of calcified skeletons. With this technique, Lafuste and Tourneur (1988) showed the microlamellar microstructure of the wall of $\mathrm{Fa}$ vosites gothlandicus Lamarck, 1916 from the Wenlock of Gotland and the fibrous nature of the wall of Devonian Favositids. In addition, the location of mural pores is of great interest to separate Favosites s.s. (face mural pore $=\mathrm{P}_{2}$ ) and Paleofavosites
(= Mesofavosites see Powell and Scrutton, 1978) with corner pore $\left(\mathrm{P}_{1}\right)$ and face pore $\left(\mathrm{P}_{2}\right)$.

\section{Microlamellar Favositids from the Devonian of Algeria}

The study of Devonian Favositids from the Ougarta area (Western Sahara) shows, for the first time, that microlamellar representatives of Favositids persist during the Devonian time.

Favosites goldfussi pyriformis (Lecompte, 1939 sensu Le Maître, 1952).

Studied specimen No. GFCL 452 (Le Maitre, coll. Lille), Dkhissa Formation, Upper Lochkovian-Praguian (Paris et al., 1997). Small corallum without 'epitheca', wall spinose, tabulae flat, $\mathrm{P}_{1}$ 
and $\mathrm{P}_{2}$ mural pores (figures $1 \mathrm{~A}$ and $\mathrm{B}$ ). This unusual disposition of pores for a Devonian Favositid is associated with a microlamellar microstructure of the wall.

The microlamellae studied by 'LFP' are clearly curved, rather thick and cupulate. They are larger than usual for microlamellae and their maximum diameter more than $20 \mu \mathrm{m}$ and often 25-30 $\mu \mathrm{m}$, even reaching 38-40 $\mu \mathrm{m}$ (figures 1D-F). Despite the size that some lamellae reach, their shape (outline and proportion) is typical of microlamellae.

The median 'dark' line is not built by 'plaquettes' or granules as in Favosites s.s. and Paleofavosites (Lafuste and Tourneur, 1988) but by elongated fiber-like units more or less in a fan setting (figure 1C).

\section{Favosites goldfussi saourensis Le Maître 1952.}

Numerous specimens from the lower part of the Chefar el Ahmar Formation, late Emsian (Plusquellec coll. Brest). Corallum hemispherical with 'epitheca', wall thickened on the lower side (figures $2 \mathrm{~A}$ and $\mathrm{B}$ ), tabulae flat, $\mathrm{P}_{1}$ and $\mathrm{P}_{2}$ mural pores. Stereoplasm of the wall microlamellar, median line fiber-like (figure $2 \mathrm{C}$ ). As in the former species the microlamellae are large (maximum diameter mostly between 22 and $44 \mu \mathrm{m}$ ).

\section{Species of other localities}

Beaumontia guerangeri Milne-Edwards and Haime, 1851 sensu Huffner, 1917, Lower Devonian of Bithynia (Turkey).

The specimens of Huffner are microlamellar (one of them is close to $F$. goldfussi saourensis) and cannot be assigned to $B$. guerangeri which is a Praemicbelinia.

Mesofavosites scbouppei Potthast and Oekentorp, 1987, Upper Emsian-Lower Eifelian of Hamar Laghdad (Marocco).

This species recently re-assigned to Striatoporella by Oekentorp and Tong-Dzuy (1996) shows a microlamellar stereoplasm (diameter of microlamellat up to $35-50 \mu \mathrm{m}$ ) and a fiber-like dark line; the large and numerous spines are fibrous (figure 3C).
Favosttes bobemtcus Maurer 1896 and subspecies, Lower Devonian of Bohemia, Germany and Algeria.

All the studied specimens of this common species and of subspecies $F$. bobemicus mosellanus (Weissermel, 1941) and ' $F$ ' bobemicus grandis (Le Mâttre, 1952) show microlamellar walls and pores in $P_{1}$ or $P_{2}$ positions.

\section{Generic and specific discussion}

They are no major differences between the microlamellar Ordovician and Silurian Favositids and the Devonian ones except the size of microlamellae and the fiber-like lay-out of the median line.

In our opinion the morpholngy of the microlamellae is not due to diagenesis and we consider that the evolution of microlamellar Favositids is characterized by (i) an increase of the size of these biocrystals ('large microlamellae') and (ii) the substitution of granules of the median dark line by fiber-like units.

The species described here are provisionally assigned to the genus Paleofavosites.

\section{A long-lived lineage}

New data about Devonian Favositids from Northern Gondwana and south Laurussia show:

(i) the record of microlamellar lineages up to the Lower Eifelian;

(ii) the development of fiber-like units in the median line and the increase of the size of microlamellae giving rise to 'large microlamellae:

(iii) the presence of Paleofavosites-identified both by structural and nicrostructural features-in the Devonian;

(iv) the confinement of Favosites s.s. in the Ordovician and Silurian;

(v) the high frequency of the microlamellar Favositids in the Devonian.

\section{Introduction}

Tabulés et Tétracoralliaires, associés aux Stromatopores, contribuent largement à l'édification des écosystèmes récifaux, en particulier au Silurien (Wenlock) et au Dévonien moyen (Copper, 1989). Les taxons étudiés ici ne sont pas nécessairement liés à ces faciès particuliers (c'est le cas des espèces de l'Ougarta) mais tous, néanmoins, se rencontrent à des latitudes comprises entre $10^{\circ} \mathrm{S}$ et $40^{\circ} \mathrm{S}$ (base paléogéographique Scotese et Golonka, 1992).

Le genre Favosites Lamarck 1816 peut être considéré comme l'archétype des Tabulés. Ses caractères morphologiques très simples expliquent les innombrables citations de ce genre, de l'Ordovicien au Permien. Aussi, les indications fournies par la microstructure sont-elles précieu- ses pour délimiter ce vaste ensemble et y établir des subdivisions. Dès 1962, Lafuste a reconnu une disparité microstructurale entre les formes du Dévonien et celles du Silurien ; ces recherches ont été poursuivies par les Soviétiques (Mironova, 1974 ; Smirnova, 1974 ; Yanet, 1974).

L'usage des lames ultra-minces à faces polies (Lafuste, 1970) — dites LFP en français - a permis d'améliorer considérablement l'observation des caractères microstructuraux. C'est par cette méthode que Lafuste et Tourneur (1988) ont fixé la constitution microlamellaire du type de Favosites gothlandicus Lamarck 1816, du Wenlockien de Gotland, et d'autres Favositides siluriens d'origines diverses. Ils ont alors souligné la nature fibreuse des Favosites dévoniens et les implications systématiques de cette différence. Des observations nouvelles obligent à 
nuancer ce modèle de répartition stratigraphique quelque peu simplificateur.

II faut également rappeler que la position des pores muraux a conduit à séparer de Favosites Lamarck 1816 (pores localisés sur les faces des murailles), Paleofavosites Twenhofel 1914 (pores restreints aux angles des corallites) et Mesofavosites Sokolov 1951 (pores situés à la fois sur les faces et les angles des corallites). Powell et Scrutton (1978) ont montré pour ces deux derniers genres qu'il fallait tenir compte d'une variation considérable de ces deux caractères et en faire un usage circonspect.

\section{Favositides microlamellaires du Dévonien d'Algérie}

Si les observations microstructurales de Mironova (1974) et Yanet (1974) sont confirmées (en l'absence de lames LFP, il peut y avoir confusion entre les fibres et les empilements de microlamelles), les premiers Favositides fibreux apparaissent dès le Ludlow, mais, contrairement au schéma en usage, la lignée des formes microlamellaires persiste au Dévonien. Deux exemples bien documentés du Dévonien d'Agérie le montrent.

\section{Favosites goldfussi pyriformis Lecompte 1939 sensu Le Maître 1952}

La colonie étudiée, GFCL 452 (matériel Le Maître), provient du Grès de Dkhissa des Monts d'Ougarta (Algérie), d'âge Lochkovien supérieur-Praguien (Paris et al., 1997).

Le polypier de petite taille, pyriforme, sans « épithèque ", possède des calices inégaux, une muraille épineuse, des planchers complets, plans ou légèrement cuncaves (figure 1A). Les pores muraux sont situés sur les faces des corallites, sur une ou deux files (pore de type $P_{2}$ ou pore pariétal), mais aussi aux angles des corallites (type $P_{1}$ ) caractère typique des genres Paleofavosites-Mesofavosites (cf. Powell et Scrutton, 1978) longtemps considérés comme uniquement ordoviciens et siluriens (figure 1B). Cette structure que l'on peut considérer comme archaïque est associée à un autre caractère primitif : la nature microlamellaire de la muraille.

Les lames d'épaisseur pétrographique (30 um) montrent une muraille constituée d'une lame médiane sombre flanquée d'une couche claire peu lisible, la stéréozone. En lame LFP (2-5 $\mu \mathrm{m})$, la stéréozone présente une constitution microlamellaire (figure 1C-F).

Les microlamelles souvent trapues et relativement épaisses ont une forme arquée très nette ; leur aspect est le même en coupes transversale et longitudinale; ce sont des microlamelles cupulaires. La convexité de leur face proximale (côté lame médiane) est généralement plus accentuée que la concavité de la face distale ; la cupulation est fortement marquée sur les deux faces. Elles sont, de part et d'autre de la lame médiane, opposées dos à dos, et leur côté concave fait face au polype. Quelques microlamelles allongées ont un aspect atypique. L'épaisseur la plus fré- quente des microlamelles est de 5-7 $\mu \mathrm{m}$, la hauteur de 10-15 $\mu \mathrm{m}$ et la longueur (ou le diamètre) est fréquemment supérieure ou égale à $20 \mu \mathrm{m}$, parfois $25-30 \mu \mathrm{m}$, voire $38-40 \mu \mathrm{m}$.

La lame médiane montre une organisation plus complexe que chez Favosites s.s. et Paleoravosites; les plaquettes décrites chez ces deux genres (Lafuste et Tourneur, 1988) sont remplacées par des biocristaux aciculaires organisés en bouquets divergents bien visibles sur les coupes longitudinales; il y a fibrisation de la lame médiane (figure 1C). Les épines quant à elles sont fibreuses.

\section{Favosites goldfussi saourensis Le Maître 1952}

Nombreuses colonies provenant du niveau coralligène situé à la base de la Formation de Chefar el Ahmar dans les Monts d'Ougarta (Algérie), Emsien supérieur terminal.

Polypier de petite taille, discoïde à hémisphérique, face proximale à "épithèque ", calices inégaux à paroi épaissie en bordure de colonie, épines présentes, planchers complets, plans (figure $2 A-B$ ). Pores muraux de type $P_{1}$ et $\mathrm{P}_{2}$ souvent obturés par des diaphragmes $(\mathrm{Pp})$. La muraille est microlamellaire, la lame médiane apparaît sombre et continue sur les lames d'épaisseur pétrographique. Les microlamelles présentent les mêmes caractéristiques que dans l'espèce précédente. Leur épaisseur la plus fréquente est de 6-9 $\mu \mathrm{m}$, leur hauteur de 10-18 $\mu \mathrm{m}$ et leur longueur, très variable, est dans la plupart des cas comprise entre 22 et $44 \mu \mathrm{m}$ (figure $2 \mathrm{C}-\mathrm{F}$ ). On atteint là, pour ce dernier paramètre, des dimensions qui sont généralement celles des lamelles, dont le diamètre est souvent compris entre 30 et $60 \mu \mathrm{m}$, mais pcut aussi dćpasser les $100 \mu \mathrm{m}$. Toutefois, les caractéristiques morphologiques des biocristaux de $F$. goldfussi saourensis sont indubitablement celles des microlamelles, épaisseur importante dans la partie centrale, convexité très affirmée de la face proximale, cupulation très marquée et organisation des biocristaux en ensembles séparés par des lignes de rebroussement.

La lame médiane est constituée comme chez F. goldfussi pyriformis sensu Le Maître d'éléments aciculaires légèrement divergents. Les épines sont fibreuses.

\section{Autres lieux, autres espèces}

\section{Beaumontia guerangeri Milne-Edwards et Haime 1851 sensu Huffner 1917 (Dévonien inférieur de Bithynie, Turquie) \\ Les trois colonies déterminées comme telles sont des formes microlamellaires, dont l'une est à rapprocher de $F$. goldfussi saourensis Le Maître (colonie hémisphérique, muraille microlamellaire, planchers complets, pores $P_{1}$ et $P_{2}$ ). Elles ne présentent aucune relation de parenté avec $B$. guerangeri espèce-type du genre Praemichelinia Lafuste et Plusquellec 1980.}

\section{"Mesofavosites " schouppei Potthast et Oekentorp 1987 \\ Un représentant de ce genre réputé Ordovicien-Silu- rien (Hill, 1981, p. F 547) est décrit dans l'Emsien supé- rieur-Eifelien inférieur d'Hamar Laghdad, Maroc. La dé-}




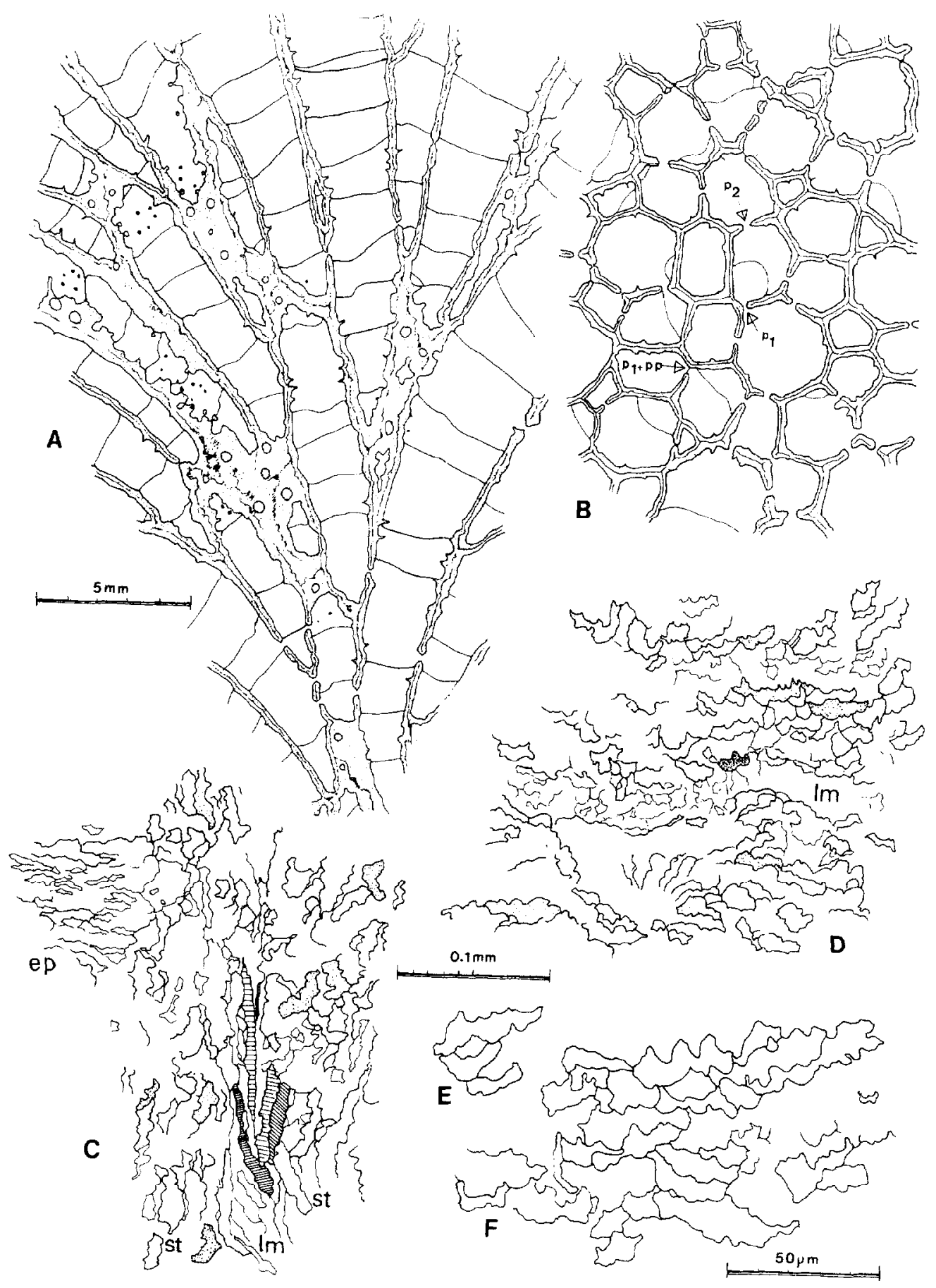

Figure 1. Favosites goldfussi pyriformis Lecompte 1939 sensu Le Maître 1952, Spécimen GFCL. 452 ; coll. Le Maître, Faculté libre des Sciences, Géologie, Lille, France. A. Coupe longitudinale (lame GFCL 452b). B. Coupe transversale, $P_{1}$ : pore d'angle, $P_{2}:$ pore pariétal (lame GFCL $\left.452 a\right)$. C. Coupe longitudinale dans la muraille, Im : lame médiane avec certains cristaux hachurés, st : stéréozone, microlamelles caractéristiques en pointillé, ep : épine fibreuse ( $L F P$ GFCL 452e). D. Coupe transversale dans la muraille, (LFP GFCL 452C). E. Coupe transversale, contour de quelques microlamelles de la muraille (LFP GFCL 452c). F. Coupe longitudinale, contour de quelques microlamelles (LFP GFCL 452e).

Favosites goldfussi pyriformis Lecompte, 1939 sensu Le Maitre, 1952, Specimen GFCL 452; coll. Le Maitre, Faculté Libre des Sciences, Géologie, Lille, France. A. Longitudinal section (thin section GFCL 452b). B. Transverse section, $P_{1}$ : corner pore, $P_{2}$ : face mural pore (thin section $G F C L 452 a$ ). C. Longitudinal section in the wall, Im: median 'dark' line with some hatched fibers, st: stereozone, dotted microlamellae, ep: fibrous spine ('LFP'-for ultra thin section with polished laces-CFCL 452e). D. Transverse section ('LFP' GFCL 452C). E. Transverse section in three microlamellae of the wall ('LFP' GFCL 452c); F. Longitudinal section in some microlamellae of the external half part of the stereozone of the wall ('LFP' GFCL 452e). 

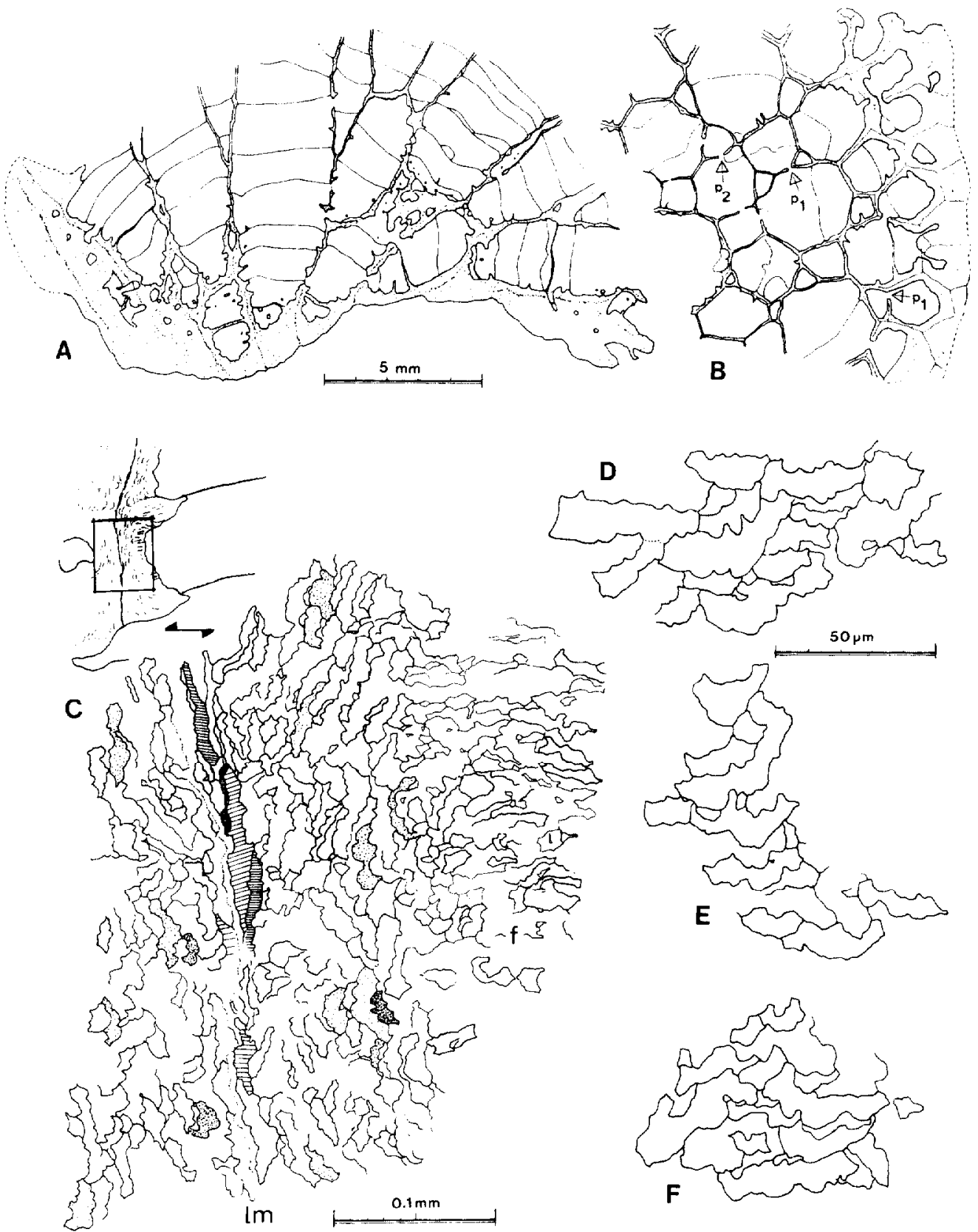

Figure 2. Favosites goldfussi saourensis Le Maître 1952. Spécimen LPB 17287, coll. Faculté des Sciences, Paléontologie, Brest, France. A - Coupe longitudinale; remarquer l'épaississement caracléristique de la muraille face proximale (empreinte à l'acétate L.PB 17287h). B. Coupe transversale, $P_{1}-P_{2}$ idem figure 1 (lame LPB 17287i). C. Coupe longitudinale dans la muraille au voisinage d'une épine (cf. schéma annexe), éléments aciculaires de la lame médiane en hachures, $f$ : la stéréozone microlamellaire est localement recouverte d'une couche de fibroïdes (LFP LPB 17287g). D. Coupe longitudinale, contour de quelques microlamelles (LFP 17287g). E et F. Coupes transversales (LFP LPB 17287f).

Favosites goldfussi saourensis Le Maître, 1952. Specimen LPB 17287, coll. Faculté des Sciences, Paléuntulugie, Bresl, Framce. A. Longitudinal section, note the thickening of the wall at the proximal side of the corallum (acetate peel $L P B 17287 \mathrm{~h}$ ). B. Transverse section, $P_{7}-P_{2}$ as in figure 1 (thin section $L P B$ 17287i). C. Longitudinal section in the wall in the vicinity of a spine (cf. associated sketch), acicular elements of the median 'dark' line hatched, dotting on some microlamellae, f: fibroids in some place on the external side of the microlamellar stereozone ('LFP' $L P B 17287 \mathrm{~g})$; $\mathbf{D}$. Longitudinal section, outline of some microlamellae ('LFP' $L P B 17287 \mathrm{~g}$ ). E, F. Transverse sections ('LFP' $L P B 17287 f$ ).

termination est principalement basée sur la localisation des pores muraux (figure $3 \mathrm{~A}$ ), et les auteurs considèrent que l'aspect fibreux de la stéréozone est d'origine diagénétique. Des lames LFP réalisées dans du matéricl paratypique montrent en fait une stéréozone constituée de microlamelles, pouvant atteindre $35-50 \mu \mathrm{m}$ de diamètre (figure $3 C$ ), de très belles épines fibreuses largement recouvertes sur leur lace supérieure par les microlamelles, et une fibrisation de la lame médiane, au moins dans la zone périphćrique des colonies. Dans une note récente, Oekentorp et Tong-Dzuy (1996) réattribuent l'espèce au genre Striatoporella Rukhin 1938. 

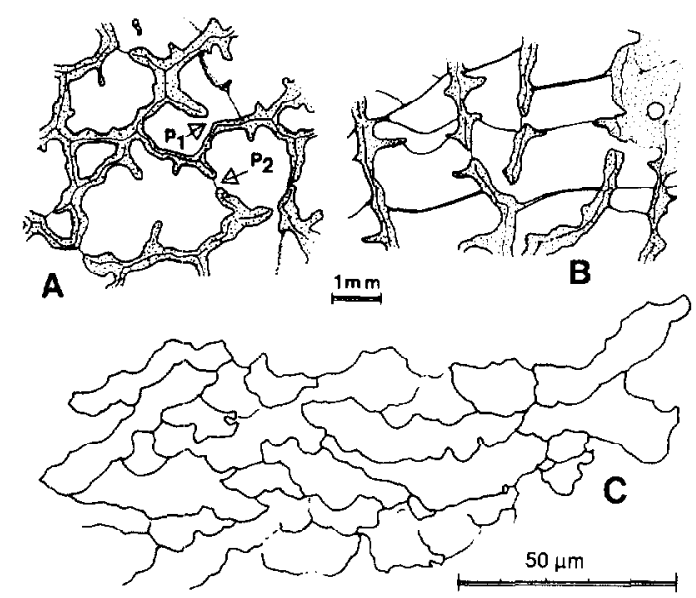

"Favosites » bohemicus Maurer 1896 et sous-espèces

Un groupe un peu hétérogène rassemble des Favositides à grands polypiérites du Dévonien supérieur, autour de l'espèce "Favosites" bohemicus Maurer, commune en Bohême et dans certains faciès hercyniens d'Allemagne; on y compte "Favosites " bohemicus mosellanus Weissermel 1941 de Rhénanie et "Favosites " bohemicus grandis Le Maître 1952 de l'Ougarta, Algérie. La systématique spécifique de ce groupe reste à revoir. Toutes les colonies que nous avons pu examiner, provenant de Bohême, d'Allemagne et d'Afrique du Nord, montrent des parois à constitution microlamellaire comparable à celle décrite par aillcurs dans la préscnte notc. Pores pariétaux et angulaires coexistent dans les sections.

\section{Attribution générique et spécifique}

Aucune différence essentielle ne semble distinguer les Favositides microlamellaires du Dévonien de ceux de I'Ordovicien supérieur et du Silurien, la forte taille des microlamelles et la différenciation de la lame médiane mises à part.

Les dimensions élevées des microlamelles ne paraissent pas dues à des phénomènes diagénétiques, dans la mesure où ces cristaux présentent généralcment une seule courbure principale, pas de dichotomie et une cupulation du même type que celles des microlamelles standard.

Nous interprétons donc nos observations en terme d'évolution du tissu minéralisé, se traduisant au Dévonien par une augmentation de la taille des microlamelles (pour lesquelles nous utiliserons le terme de grandes microla-

\section{RÉFÉRENCES}

Copper P. 1989. Enigmas in Phanerozoic reef development, Mem. Ass. Australas. Palaeontols, 8, 371-385

Hill D. 1981. Part F. Coelenterata, Suppl. I. Rugosa and Tabulata, in : Moore R.C. et Teichert C. (éds.), Treatise on Invertebrate Paleontology, F1-F762

Hüffner E 1917. Beiträge zur Kenntnis des Devons von Bithynien. Jahr. Königl. Preuss. Geol. Land. XXXVIII (2), 260-357
Figure 3. “ Mesofavosites » schouppei Potthast et Oekentorp 1987. Specimen $P_{1}-$ MA-97 (Paratype) ; coll. Geologisch-Paläontologisches Institut, Münster, Allemagne. A. Coupe transversale (lame $P_{1}-M A-97-1$ ). B. Coupe longitudinale (lame $\left.P_{1}-M A-97-1\right)$. C. Coupe transversale, contour des microlamelles (lame LFP $P_{1}-M A-97-4$ ).

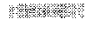

Mesofavosites' schouppei Potthast and Oekentorp 1987. Specimen $P_{1-}$ MA-97 (Paratype); coll. Geologisch-Paläontologisches Institut, Münster, Germany. A. Transverse section (thin section $P_{1}-M A-97-1$ ). B. Longitudinal section (thin section $\left.P_{7}-M A-97-1\right)$. C. Transverse section, outline of some microlamellae ('LFP' $\left.P_{7}-M A-97-4\right)$.

melles) et par une tendance à la fibrisation de la lame médiane; ce dernier phénomène est commun chez les Tabulés, soit en fin de lignée, soit au cours de la croissance (Lafuste et Plusquellec, 1985 ; Lafuste et Tourneur, 1991).

Nous rapportons provisoirement les "Favosites" à grandes microlamelles, lame médiane "fibreuse", pores $P_{1}$ et $P_{2}$ au genre Paleofavosites, mais la création d'un nouveau sous-genre est envisageable. $L$ 'analyse des déterminations spécifiques dépasse le cadre de cette note, mais il est clair que l'attribution des Favositides de l'Ougarta à l'espèce goldfussi ne peut être maintenue. Cette forme possède une lame médiane granulaire, des parois fibreuses et des pores localisés sur les faces des corallites.

\section{Une lignée persistante}

Les données nouvelles concernant quelques Favositides du Dévonien du Nord Gondwana et du Sud de la Laurussia montrent :

- la persistance des lignées microlamellaires jusqu'à l'Eifelien inférieur ;

- une évolution de la lame médiane et de la taille des microlamelles ;

- l'extension dévonienne du genre Paleofavosites, défini à la fois sur des critères structuraux et microstructuraux ;

- la localisation - dans l'état actuel des connaissances - du genre Favosites s.s. de l'Ordovicien supérieur au Silurien ;

- le caractère non exceptionnel de ces formes.

Lafuste J. 1970. Lames ultra-minces à faces polies. Procédé et application à la microstructure des Madréporaires fossiles, $C . R$. Acad. Sci. Paris. 270, série D, 679-681

Lafuste J. et Plusquellec Y. 1985. Structure et microstructure de quelques Michelinidae et Michelinomorphes (Tabulata paléozoïques), Bull. Mus. nat. Hist. nat. Paris, 7 (C/1), 13-55

Lafuste $\mathrm{J}$, et Tourneur F. 1988. Microstructure du genre Favosites Lamarck 1816 (Tabulata) et de Favositides du Silurien, avec une révision du néotype de Favosites gothlandicus Lamarck 1816. Ann. Soc. géol. Belgique, 110, 189-198 
Lafuste J. et Tourneur F. 1991. Microstructure du genre Acaciapora Moore and Jeffords 1945 (Tabulata; Pennsylvanien de I'Oklahoma, USA), Geol. Palaeont., 25, 99-105

Le Maître D. 1952. La faune du Dévonien inférieur et moyen de la Saoura et des abords de l'Erg el Djemel (Sud-Oranais), Mat. Carte géol. Algérie, Paleont., 12, 1 170

Mironova N.V. 1974. Tabulés éodévoniens de l'Altaï et du Salair, Tr. SNII GGMS, 163, 1-166 (en russe)

Oekentorp K. et Tong-Dzuy Thanh. 1996. Remarks on the genero Striatoporella and 'Riphaeolites' (Devonian Tabulata), N. Jb Geol. Paläont. Mh., 5, 293-308

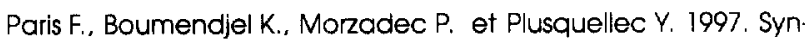
thèse chronostratigraphique du Dévonien de l'Ougarta (Sahara occidental, Algérie), Ann. Soc. géol. Nord, 5, 117-121

Potthast I. et Oekentorp K. 1987. Eine Favositiden-Fauna aus dem
Emsium/Eifelium des Hamar Laghdad, Tafilalt (SE-Marokko). Münst. Forsch. Geol, Palöont., 66, 57-94

Powell J.H. et Scrutton C.T. 1978. Variations in the Silurian tabulate coral Paleofavosites asper, and the status of Mesofavosites. Palaeontology, 21 (2), 307-319

Scotese R. et Golonka J. 1992. Paleogeographic atlas. Paleomap project. Univ. Texas, Mobil Explor. Produc. Serv.

Smirnova M.A. 1974. On the wall microstructure of Late Silurian and Early Devonian Favositdae, in : Sokolov B.S. (éd.). Ancient Cnidaria, Akad. Nauk SSSR, Sibirsk. Otdel., Tr. Inst. Geol. Geofiz., $201,80-86$

Yanet F.I. 1974. Parallelism in the development of Silurian and Devonian Tabulata of the Urals, in : Sokolov B.S. (éd.). Ancient Cnidaria, Akad. Nauk, SSSR, Sibirisk. Otdel., Tr. Inst. Geol. Geofiz., $201,122-128$ 